\title{
A NOTE ON THE MODERN TECHNIQUE OF POSTERIOR VAGINAL CGELIOTOMY.
}

\author{
By E. HASTINGS TWEEDY, F.R.C.P.;
}

Ex-Assistant Master, Rotunda Hospital.

[Read in the Section of Obstetrics, November 22, 1901.]

THE specimen I have the honour of presenting to your consideration this evening consists of the right Fallopian tube and a parovarian cyst removed from a very stout patient of mine in Steevens' Hospital, by the operation known as Prior's posterior vaginal coliotomy. Prior's operation does not appear to have received the attention it deserves either in this country or yet in England, and it is for the purpose of eliciting the views of members here to-night concerning it that I now present this very commonplace specimen. With a few exceptions, Irish surgeons seem to have made incision into the anterior fornix their operation of election when performing colpotomy, and there does not appear any indication that the operation is advancing in popular favour, or even holding its own.

The objections urged against it are, that the limited opening into the abdominal cavity does not permit of the various steps of the operation being seen, consequently irreparable harm may be inflicted on important structures, which neither the sense of touch nor a previous knowledge of anatomy can safeguard.

I believe we possess in Prior's operation a means of overcoming these and other objections to a considerable extent, provided conditions be favourable; or, in other words, provided a woman has borne a full-term child, and the vagina be not inflamed or abnormal. 


\section{Technique of Posterior Vaginal Coliotomy.}

In the removal of this tumour I followed his procedure, which I shall now describe in detail. The patient, a very stout woman, with a roomy vagina, was prepared as for an ordinary abdominal section, anæsthetised, and then placed on Burndt's operating table, in the usual dorsal position. The shaved vulva and the vagina were now for the second time scrubbed thoroughly with soap and water (green soap is to be preferred), then douched out with creolin solution, and finally bathed in an alcoholic solution of biniodide of mercury (1 in 1000). This latter antiseptic is the one now most relied on in Steevens' Hospital for the final stages of skin disinfection. It has not alone the merit of great penetrability, but likewise hardens and cornifies epithelium, so hindering maceration and its removal during the operation. A bullet forceps was then attached to the posterior cervical lip, and the fornix exposed by means of a Martin's speculum. On moring the cervix up and down, the crescentic fold, which indicates the reflection of the vagina on to the cervix, was clearly made out. This I picked up by a forceps, and incised the vaginal mucous membrane to the extent of about an inch, in the transverse direction, by means of sharp-pointed scissors. This incision did not go deeper than the mucous membrane. A catch forceps was then fixed on the posterior flap, and my assistant was directed to make firm traction on this, as well as on the foreeps attached to the cervix. My index fingers were now inserted into the wound, and tore with ease through the peritoneum, close to its uterine reflection. Before withdrawing the fingers the wound was enlarged by lateral tearing. This procedure is easily carried out, and does not involve fear of injury to the ureters, nor to the uterine vessels, provided undue force be not employed. The fingers having been withdrawn, a gauze wipe wrung out in saline solution, and to which a piece of silk is attached for ease in its removal, was now placed in the abdominal cavity to collect blood or other 
discharge, and to partially shut off the intestines from dust infection.

The next step of the operation consisted in the passing of two long vaginal retractors into the wound, and the removal of all other instruments from the vagina. The table was now lowered, so that the patient, with her knees still in the gynæcological crutches, assumed the "head downward" or Trendelenburg position. On now widely separating the retractors the intestines could clearly be observed as they slowly receded from the field of operation, and an excellent view of the cyst-in size about that of a billiard ball-was obtained. The anterior blade was now removed, and the tumour, seized by a couple of fingers, presented no difficulties in its removal. The patient, raised again to the horizontal position, had the temporary pad removed, a strip of moist iodoform gauze was inserted just within the lips of the wound, and the vagina well packed around the fornices with similar material. This packing was taken out on the third day, the patient being under the influence of chloroform, and lying in the Sims' position. A new one was inserted, but not through the wound, this being a departure from the original operation, which provides for a drainage for seven days-an unnecessary precaution to my mind.

Dilatation of the sphincter and the passage of a catheter every four hours are further suggestions the soundness of which will not be called in question.

The operation as thus described is a simple and rapid procedure, and remarkably free from complications. It is obvious that it possesses a wide range of usefulness, but as the indications for its employment are now sufficiently well known I need not dwell on them. As a means of gaining access to, and a greater knowledge of, the structures on which we intend to operate, I believe it to be far superior to the older operations. With it injuries to the rectum should be avoided, while ad- 


\section{Technique of Posterior Vaginal Coeliotomy.}

hesions can be attacked, and separated with as much ease, and with far less danger, than if dealt with by means of a suprapubic incision. Moreover, should the bowel give way while endeavouring to combat adhesions, the accident is not likely to prove of a very formidable character. The general peritoneal cavity is almost certainly shut off from infection, and the rupture, even though unclosed at the time of operation, will heal spontaneously in the majority of instances. I hold that all recent cases of pyosalpinx, which call for operative interference, should, when possible, be approached through the vagina. Multiple incisions into the abscess cavities, with subsequent drainage-the whole procedure, guided by sight and touch, even though it fail to effect a permanent cure-will at least render any more radical operation afterwards undertalken comparatively safe.

I trust I shall not be understood as saying that the operation just described marks any radical departure in vaginal coeliotomy. I am aware that posterior colpotomy has been performed very frequently by different surgeons in this city who can testify to the easy manner in which tubes and ovaries, even though normally situate, can be palpated and directly examined by its means. My contention merely is that Prior's operation makes a step forward in its technique, and somewhat enlarges its scope. Furthermore, it is obvious that anterior colpotomy must of necessity remain the proper operation under certain conditions. Finally, I should like to point out the great advantage afforded by modern operating tables, giving as they do a good Trendelenburg position, without which Prior's operation becomes impossible of execution. And of scarcely less importance is a sufficiency of light, hardly procurable in any but the recently built operating theatre, of which we possess a very good example in Steevens? Hospital. 
DR. MACAN remarked that he was old enough to confess he did not know what Prior's operation is, and was not much enlightened by the paper. Operators had long used both anterior and posterior colpotomy, and Prior did nothing, in his opinion, except the posterior operation with the use of the Trendelenburg position. He did not think the position in such an operation brings the parts well into view-better without it; nor could he understand how the ureters or vessels could come into the way of the operator in posterior colpotomy. He looked upon the removal of plugs as almost always painful unless you wait for the presence of some moisture-a course to be recommended-always supposing there is no rise of temperature. In all such cases the question naturally arisesWhat route will the surgeon pursue: vaginal or abdominal ? In unilateral diseases he preferred the superior operation, as one gets a better view of the condition of the parts. In cases of abscess the vaginal route is, as a rule, preferable, and when tumour is located in Douglas's pouch posterior colpotomy is to be preferred.

DR. PUREFoY did not think that posterior colpotomy is so free from all risks as the paper might lead hearers to think. In two recent cases he had had secondary bleeding. One case occasioned him some anxiety, as the bleeding came on during the night, and he found on his arrival a vessel spouting at the angle of the wounds on which he had to apply a catch forceps.

DR. ALFRED SMITH was of opinion that with the use of antiseptics opening the peritoneum has been robbed of its terrors, and he uses the abdominal route in all cases, as giving the best view of the parts, and because it is no more liable to shock than the vaginal. The painful process of removing plugging might be avoided if a bag were used, and this bag filled with tampons of small plugs, which could be readily removed without causing pain. He had found the early removal of plugs comparatively painless, and had no bad results from doing so.

DR. JELLETT said the Section had wandered from the subject of Dr. Tweedy's paper, which was a description of Prior's operation to be used in suitable cases. It is obvious, he remarked, that if adhesions were present no tissues fall down, and there is no increased area of view. Prior's operation was useful in cases of purulent bulging.

DR. WILliam SmyLY could not understand Dr. Jellett's objection. How can we discuss the operation other than by comparing it 
with the methods of other operators? An operation very similar to this of Prior's is recommended in cases of hysterectomy for cancer. The Trendelenburg position had the great advantage of allowing of plugging the wound. It may be necessary to allow plugs to remain in a long time. As a whole, he prefers the abdowinal route, yet he confessed there are occasions when the ragina is preferable. Our views are modified by time. We are not now so anxious to excise everything. The tendency is to look at patients years after operation, and on their then condition to decide on the benefits or otherwise of the procedure. Years ago he removed a unilateral pyosalpinx, and did it by the abdominal route; he was now able to record that the patient, who went to the country, has been for years quite well, and the mother of a healthy child. In one case he removed the ovaries by the vaginal route, leaving a little of the tubes. Years afterwards the patient had to be again operated on; the remaining portions of the tubes were removed, and the patient is now quite well. Had he selected the abdominal route the second operation would not have been necessary.

DR. TWEEDY, in reply, said that he had brought Prior's operation under notice because it was little known, and, in certain conditions, would be found useful. 